

\title{
EL CRECIMIENTO DE UNA SEMILLA COMO OPORTUNIDADDE APRENDIZAJE EN CASA PARA NIÑOS CON HABILIDADES DIFERENTES
}

\section{The Growth of A Seed as a Home Learning Opportunity for Children With Different Abilities}

\section{A germinação de uma semente como uma oportunidade de aprendizagem em casa para crianças com diferentes habilidades}

\author{
Milagros Esperanza Rojas Salgado*
}

Fecha de recepción: 19 de septiembre de 2018

Fecha de aprobación: 18 de abril de 2019

\section{Resumen}

El presente artículo corresponde a un estudio de caso en relación con la experiencia educativa domiciliaria llevada a cabo con Nicolás, un niño de seis años con características diferentes que se tipificaron principalmente en un diagnóstico de Trastorno del Déficit de Atención con Hiperactividad (TDAH), con predominio de desatención y un Trastorno Específico del Lenguaje (TEL), fundamentalmente con dificultades en la comprensión del lenguaje verbal. Este artículo tiene como propósito principal documentar la experiencia pedagógica realizada con Nicolás en relación con el aprendizaje de las características de las plantas y el promover en él el desarrollo de diversas capacidades orientadas al pensamiento científico, como la observación, el planteamiento de preguntas e hipótesis, la comprobación de estas hipótesis mediante experiencias, el registro de la observación, la elaboración de conclusiones y el planteamiento de nuevas preguntas, que nuevamente daban inicio al proceso de indagación. El lenguaje verbal, no verbal y la escritura fueron privilegiados y potenciados en cada uno de los procesos anteriormente enunciados. Metodológicamente, el presente artículo es de corte descriptivo en el cual se empleó la observación y la documentación de evidencia gráfica como técnicas de recolección de datos. A partir del presente caso se concluye que las actividades pedagógicas en las ciencias naturales simbolizan una oportunidad privilegiada para lograr aprendizajes propios no solo de esta área del conocimiento, sino también aquellos aprendizajes necesarios e inherentes a las características diferentes de niños como el que se documenta.

Palabras clave: aprendizaje; enseñanza; microenseñanza; atención; necesidades educacionales

* Estudiante de doctorado en psicología de la Universidad Nacional Mayor de San Marcos (Perú).

Correo electrónico: milagros.rojas@unmsm.edu.pe 


\section{Abstract}

This paper corresponds to a case study in relation to a home educational experience carried out with Nicolás, a 6-year-old boy with different abilities that were typified mainly in a diagnosis of Attention Deficit Hyperactivity Disorder (ADHD) in the predominance of inattention area and a Specific Disorder of Language (TEL), fundamentally with difficulties in verbal language understanding. The main purpose of this article is to document the pedagogical experience carried out with Nicolás in relation to the learning process about the characteristics of plants and the development of diverse scientific capacities such as observation, asking questions and hypotheses, the verification of these hypotheses through experiences, the observation record, the elaboration of conclusions and asking new questions to start the process of inquiry again. Verbal language, nonverbal one, and writing were privileged and enhanced in each of the previous processes. Methodologically, this paper is descriptive, in which the observation and documentation of graphic evidence was used as data collection techniques. From the present case, it is concluded that the pedagogical activities in the natural sciences symbolize a privileged opportunity to achieve learning outcomes not only in this area of knowledge, but also those learnings necessary and inherent to the different characteristics of children such as the one documented.

Keywords: learning; teaching; micro teaching; attention; educational needs

\section{Resumo}

Este artigo corresponde a um estudo de caso em relação à experiência educacional domiciliar realizada com Nicolás, um menino de seis anos com características diferentes, tipificado principalmente no diagnóstico de Trastorno de Déficit de Atenção e Hiperatividade (TDAH), com predominância de desatenção e Transtorno Específico da Linguagem (TEL), fundamentalmente com dificuldades na compreensão da linguagem verbal. O objetivo principal deste artigo é documentar a experiência pedagógica realizada com Nicolás em relação ao aprendizado das características das plantas e promover nele o desenvolvimento de várias capacidades orientadas ao pensamento científico, como a observação, a formulação de perguntas e hipóteses, a verificação dessas hipóteses por meio de experiências, o registro da observação, a construção de conclusões e a elaboração novas perguntas, que mais uma vez iniciaram o processo de investigação. Linguagem verbal e não verbal e escrita foram privilegiadas e aprimoradas em cada um dos processos listados acima. Metodologicamente, este artigo é descritivo, no qual a observação e documentação de evidências gráficas foram utilizadas como técnicas de coleta de dados. A partir do presente caso, conclui-se que as atividades pedagógicas nas ciências naturais simbolizam uma oportunidade privilegiada para a aprendizagem própria, não apenas nesta área do conhecimento, mas também aquelas que são necessárias e inerentes às diferentes características das crianças, como a documentada.

Palavras chave: aprendizagem; ensino; microensino; atendimento; necessidades educacionais 


\section{Introducción}

Existen ocasiones en nuestra vida personal y profesional que demandan algo totalmente diferente de aquello para lo que fuimos preparados. Una de esas situaciones es la que se nos presentan a algunos padres de familia o profesionales de la educación cuando nuestros hijos o estudiantes son identificados como niños con habilidades diferentes. Aquellos que entendemos que lo peor que puede suceder es esperar sin hacer nada (Grandin, en Hornok, 2018), nos especializamos e implementamos diversas actividades educativas para ellos. El presente escrito tiene por objetivo manifestar cómo se pueden desarrollar capacidades científicas y potenciar transversalmente el lenguaje verbal en un niño con habilidades diferentes, a través de una actividad que le produce interés genuino.

Ahora bien, si tuviera que elegir una de las características que quisiera que perdure por siempre en los niños, esta sería su curiosidad natural. También desearía que esta curiosidad se mantenga intacta frente a los "no", "no sé", "más tarde" matizados con lenguaje no verbal poco amigable al que, en su corta vida, ya se encuentran expuestos los niños. Un segundo deseo sería que puedan contar con un adulto cercano que se involucre en la fascinante labor de encontrar juntamente con ellos las respuestas a estas preguntas y, que esta búsqueda de respuestas nutra el ser curioso. Pero ¿qué significa ser curioso?

Aun siendo un término tan frecuentemente utilizado para representar alguno de nuestros estados personales, el ser curioso no goza de consenso al momento de ser definido. La bibliografía al respecto considera que ser curioso está referido a ese estado motivacional y placentero que involucra la tendencia a reconocer y buscar información o experiencias novedosas y retadoras con interés persistente (Kashdan, et ál., 2007). La curiosidad podría tipificarse, entonces, como una de las emociones que naturalmente emergen durante la infancia, en tanto que lo dinámico sería el objeto y la naturaleza de esta curiosidad, en términos de intensidad, frecuencia, involucramiento, entre otras características. Otros autores conciben a la curiosidad como una forma de motivación intrínseca y, como tal, factor indispensable en el proceso de aprendizaje.

Por otra parte, existen teorías acerca de la naturaleza de la curiosidad, algunas de ellas la conciben desde necesidades tan básicas como el hambre o la sed, mientras que otras teorías sitúan a la curiosidad como producto de la incongruencia entre una explicación construida a nivel personal sobre un objeto o evento y, lo que está pasando en un determinado momento con dicho objeto o evento, ante la existencia de una brecha de información lo suficientemente retadora. En ese sentido, Berlyne (en Rowson, 2012), identificó tres componentes de la curiosidad (Figura 1): el componente cognitivo de la curiosidad está referido a la necesidad de encontrar esa pieza de información que nos falta para completar la big picture frente a un objeto o evento; mientras que los otros dos componentes están referidos a la experiencia sensorial que tiene lugar cuando tenemos nuestro primer acercamiento a lo novedoso de ese objeto o evento.

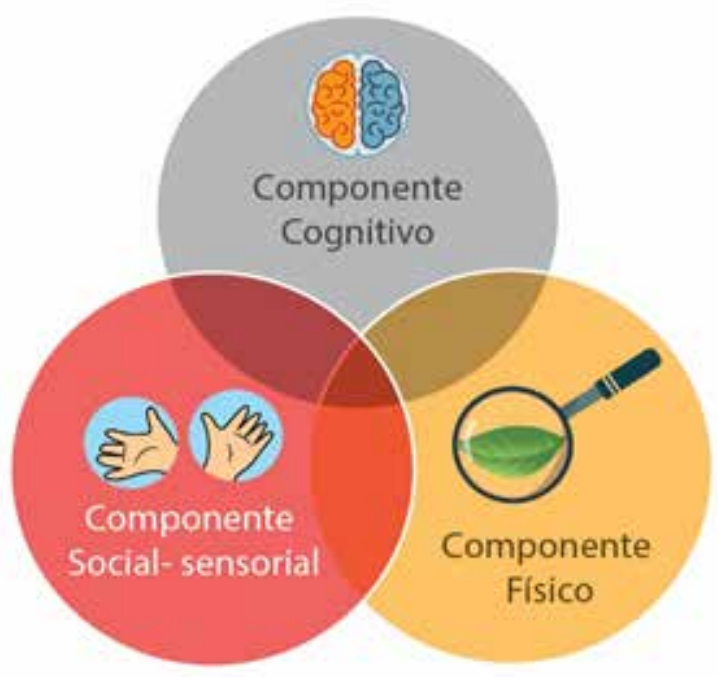

Figura 1. Componentes de la curiosidad según Berlyne

Fuente: elaboración propia

Esto es, existen determinadas características inherentes a dichos objetos o eventos que permiten causar curiosidad relacionadas con la complejidad, la novedad, la incertidumbre y el conflicto cognitivo que estos objetos o eventos supongan para nosotros. Según estén o no presentes dichas características se producirá o no curiosidad en las personas; por otra parte, la voracidad con la que, como seres humanos, demandamos constantemente de nueva información, nos define como curiosos por naturaleza. Según Kidd, et ál. (2015), la disminución de nuestra curiosidad podría ser indicador de depresión; en contraste, el ser demasiado curiosos, también podría ser un indicador de un Trastorno del Déficit de Atención con Hiperactividad (TDAH). Y ¿qué hay de malo con la relación entre tener TDAH Y ser curioso?

Cuando de ser curioso se trata no existe distinción alguna de presunciones diagnósticas ni de diagnósticos propiamente dichos. La presente historia de vida documenta cómo esta característica, al parecer, innata, juega un rol preponderante en el proceso de aprendizaje de un niño con habilidades diferentes, al que denominaremos Nicolás. 
Por otra parte, la base legal en Perú contempla principalmente tres modalidades en el sistema educativo, dichas modalidades son: Educación Básica Regular (EBR), con tres niveles, inicial, primaria y secundaria; Educación Básica Alternativa (EBA), con sus dos ciclos, inicial intermedio y avanzado; y Educación Básica Especial (EBE), con tres niveles, inicial, primaria y secundaria. Esta última modalidad se ofrece a los estudiantes que presentan multidiscapacidad y necesidades educativas especiales, asociadas a una discapacidad severa, es decir, que dependen de una persona para poder realizar actividades cotidianas. Por otra parte, el currículo de la educación básica nacional vigente considera enfoques transversales, los cuales constituyen concepciones importantes sobre las personas y las interacciones que estas establecen; dichos enfoques deben de ponerse de manifiesto en formas de actuar de los agentes educativos (Ministerio de Educación, 2016). Uno de estos enfoques es el inclusivo o de atención a la diversidad. Este enfoque se compone de valores como el respeto por las diferencias, la equidad en la enseñanza y la confianza en la persona y plantea que

\section{[...] los docentes programan y enseñan considerando tiempos, espacios y actividades diferenciadas de acuerdo con las características y demandas de los estudiantes, las que se articulan en situaciones signifi- cativas vinculadas a su contexto y realidad. (Ministerio de Educación del Perú, 2017, p. 21)}

En ese sentido, sería oportuno mencionar que Nicolás, de acuerdo con la normativa vigente, puede participar de un proceso de escolarización dentro del nivel inicial de la modalidad de la educación básica regular, pues no tipifica para la modalidad de Educación Básica Especial.

Es importante considerar que actualmente se propone la idea de neurodiversidad, término que invita a un cambio de perspectiva para entender lo positivo, la riqueza y la complejidad de la naturaleza neurológica humana, que plantea la diferencia, especialmente en procesos cognitivos asociados a pensar y aprender (Armstrong, 2015). Más allá de los planteamientos en la normativa educativa e ideas vigentes, es necesario precisar que la inclusión educativa es un derecho; sin embargo, hacerlo realidad en el campo educativo no es nada simple. No basta con la sensibilización por parte de la escuela y el estar informados de las políticas al respecto, pues para poderlas materializar en el campo de acción es imprescindible saber cómo hacerlo. Sería igual o incluso peor de desastroso aceptar a un estudiante con habilidades diferentes y luego abandonarlo a su suerte esperando a que sea él quien se "amolde" a las actividades pedagógicas usuales, eso también sería no respetar sus derechos. El cambio de paradigma que supone la educación inclusiva debe de ir acompañado, necesariamente, de cambios en la infraestructura, capacitación docente, adaptaciones curriculares, entre otros (Cerebrum, 2014).

La normativa es clara al señalar que la inclusión plantea el uso de códigos y formas de comunicación, diversificación curricular, provisión de recursos específicos y capacitación docente, para poder implementarse de manera oportuna. Lo anteriormente señalado es congruente con una de las demandas actuales del profesorado: los recursos pedagógicos y didácticos, específicamente a nivel de programas de actualización docente, para poder llevar a cabo la implementación de la tal ansiada inclusión educativa, tal inclusión educativa que, Nicolás anhela y requiere con premura. Pero que de momento, aún no es del todo posible.

Nicolás, de 6 años está "en todo a la vez". Se podría describir a Nicolás como un niño intenso, con las emociones a flor de piel, cual montaña rusa. Amante de todo lo nuevo, todo lo diferente y con una sorprendente capacidad para identificar detalles que le permiten inferir información que no puede comprender; por ejemplo, cuando una persona se expresa únicamente de manera verbal, por lo que su modo de aprender predilecto es "más hechos y gestos, menos palabras”. Nicolás es jocoso, espontáneo, creativo y manifiesta su curiosidad sin restricciones y con mucha vitalidad, características que espero se continúen manifestando también en otras etapas de su vida, pues de acuerdo con Armstrong (2015): "los niños con TDAH podrían ser parte de la vanguardia de adultos que, en esa etapa de la vida, rompen esquemas y expresan características infantiles como la imaginación, la jocosidad, la espontaneidad, vitalidad, creatividad y asombro" (p.62).

Desde el punto de vista clínico, a la edad de 1 año y seis meses, la presunción diagnóstica de Nicolás fue Trastorno del Espectro Autista, motivo por el cual la familia se involucró rápidamente en atención diversificada a nivel de terapias de interacción comunicación, juego y otras terapias alternativas que, principalmente, tuvieron como hito el reconocimiento de sí mismo, el establecimiento de contacto visual y percepción - a nivel de reconocimientode los demás.

Entre las características más resaltantes del comportamiento actual de Nicolás se encuentran que su intervalo de atención es amplio, en lo que respecta a actividades que le generan curiosidad y lo retan; no obstante en otras actividades suele distraerse con mucha frecuencia y manifestar poca persistencia al momento de realizar esfuerzos mentales, sostenidos en tareas que demandan procesos cognitivos complejos. Usa esta distracción como un mecanismo de huida en situaciones en las que no comprende 
lo que su interlocutor le está manifestando verbalmente. Nicolás necesita organizar sus tareas con apoyo de un horario diario y mediante el cual se le anticipe lo que va a acontecer en las próximas horas del día; también demanda de refuerzos verbales acompañados con claras manifestaciones no verbales a nivel de lenguaje gestual; estos refuerzos deben ser cortos, precisos y frecuentes. Nicolás suele estar en constante movimiento y expresarse de manera verbal elevando la voz. Con relación a la comprensión del lenguaje verbal, es necesario hacer uso de pictogramas y gestos como apoyos imprescindibles a modo de andamios para que infiera a partir de estos lo que se espera de él. Ante la falta de comprensión de algunas indicaciones o lenguaje figurativo, Nicolás opta por responder imitando la respuesta de algunas de las personas que comparten experiencias con él. Sorprende su buena memoria para recordar el contexto en el que se dieron estas respuestas e imitarlas en situaciones similares a las originales.

Cuando conocemos por primera vez a un niño con habilidades diferentes, frecuentemente preguntamos por el diagnóstico que tiene y, en consecuencia, orientamos las actividades pedagógicas desde una perspectiva de entender la diferencia como déficit. Para cuando empezó la experiencia con Nicolás, él tenía el diagnóstico de Trastorno del Déficit de Atención con Hiperactividad (TDAH) y Trastorno del Lenguaje (TEL), aunque los especialistas aún no eran concluyentes en retirarle la presunción diagnóstica previa de Trastorno del Espectro Autista (TEA). El orientar la experiencia que describiré en este escrito, desde la perspectiva de la valoración de las diferencias y no desde aquella que se enfoca en el déficit, me permite comentar que dadas este tipo de experiencias entre otros esfuerzos, Nicolás actualmente presenta una dinamicidad favorable en su diagnóstico.

De acuerdo con el Diagnostic and Statistical Manual of Mental Disorders (DSM), en su quinta edición, el TDAH aporta un conjunto de criterios que podrían jugar un papel desfavorecedor en el proceso de aprendizaje de niños como Nicolás; sin embargo, es interesante reconocer cómo los docentes y padres de familia podemos proponer acciones pedagógicas con el propósito de atender a las necesidades diferentes de niños como Nicolás, viendo a la diferencia no como sinónimo de inferioridad o déficit sino como oportunidad. Definitivamente este cambio de perspectiva es bastante retador, porque aun sabiendo que cada niño es único, particularmente en los niños con TDAH, esta diferencia es aún más variable, menos predecible y altamente demandante. Así, las acciones pedagógicas que se planteen son inherentes a cada niño, pues las características diferentes que significa un determinado diagnóstico se configuran de manera particular en cada caso dentro de un mismo diagnóstico.
Para los especialistas que contribuyen al desarrollo integral de Nicolás y para su familia, es más que evidente que sus necesidades educativas son particulares y deben estar planteadas de acuerdo con sus características diferentes. Las actividades de aprendizaje para él deberían considerar una clase con un número reducido de integrantes, contemplar intervalos claros de movimiento - mayores a los que normalmente se programa como parte del nivel de inicial y, un trabajo interdisciplinar que pueda potenciar las áreas de atención-, concentración, lenguaje ocupacional y aprendizaje. Lo anterior se relaciona con lo planteado por Armstrong (2015) respecto a

[...] las estrategias de aprendizaje para estudiantes con TDAH deberían considerar: darse en segmentos cortos y dinámicos, tener alto contenido emocional, tratar algún aspecto de la creatividad, activar la imaginación del niño, emplear el humor, brindar retroalimentación inmediata, relacionarse a su vida personal, utilizar situaciones novedosas, involucrar frecuentemente "cambios de estado". (p.67)

En este punto pregunto ¿acaso estas consideraciones son pertinentes únicamente para un niño como Nicolás?, seguramente el resto de la clase estaría bastante favorecida si se tuvieran en cuenta este tipo de consideraciones, además si las actividades de enseñanza se orientaran hacia un diseño cada vez más universal.

Por otra parte, la familia es una variable de suma importancia en el proceso de enseñanza-aprendizaje del estudiante. Así, actualmente se plantean modelos ecológicos del aprendizaje, los cuales ponen especial énfasis en la interacción del contexto familiar del estudiante, la escuela y las condiciones socioculturales (Centro Peruano de Audición, Lenguaje y Aprendizaje, 2019). Factores de índole familiar asociados al clima y funcionamiento del hogar, las percepciones o actitudes de los padres hacia los hijos, la implicación de la familia en el proceso educativo, así como las expectativas que se tienen respecto del futuro de los hijos, influyen significativamente en el proceso de enseñanza y aprendizaje de los niños, particularmente en aquellos en los que se identificaron dificultades específicas en el aprendizaje (Robledo, et ál., en cPAL, 2019). En ese sentido, es primordial que la familia vaya más allá de la aceptación de las características diferentes de uno de sus integrantes y se involucre actuando como un sistema de soporte para el niño, principalmente, emocional y educativo.

Hawkins, et ál. (en CPAL, 2019) identificaron algunas características deseables en las familias que actúan como ese sistema de apoyo o soporte en los estudiantes a los que me refiero, entre ellas se encuentra que estas familias valoran y alientan al proceso educativo, tratan cálidamente al niño 
y reducen las críticas -en lugar de ser autoritarias o permisivas-, tienen expectativas claras, entre otras. El contexto familiar de Nicolás se caracteriza por compartir el gusto por las ciencias naturales, el dibujo, la pintura, la música y los juegos de mesa. Además, privilegian las actividades al aire libre o los juegos de actividad motora disminuyendo al máximo el tiempo que Nicolás puede pasar frente a la televisión. Nicolás es particularmente sensible a estímulos viso-sonoros, como los fuegos artificiales, juguetes que combinan luces y sonidos al mismo tiempo, más grandes que su tamaño corporal. Por el contrario, Nicolás tiene predilección por los aromas de objetos de tocador. Se siente especialmente atraído por perfumes, jabones, champús y todo aquello que desprenda un olor agradable para sí mismo. Por otra parte, Nicolás disfruta del acercamiento hacia eventos naturales, como la luna en el cielo, el vuelo de las mariposas, los picaflores y la extracción de néctar, el desplazamiento que describe un caracol, los colores de las flores y el crecimiento de las semillas. Esos fenómenos naturales llaman poderosamente su atención y afortunadamente los disfruta en familia.

De acuerdo con Vega (2012), a la edad de cero a seis años, establecer determinantemente el momento en que el juego experimental le cede paso a la construcción del conocimiento científico es muy complejo, dado que pareciera que ambos eventos se traslaparan. Sin embargo, una de las características resaltantes de este período es que el desarrollo del lenguaje y las representaciones mentales generan el momento oportuno para desarrollar también la capacidad para plantearse preguntas sobre el mundo que rodea a los niños. A la par de lo anterior, el niño, a medida que conoce el mundo que lo rodea, estructura este conocimiento y la causalidad se objetiva y se espacializa, es decir, el niño identifica que los fenómenos que observa no necesariamente se deben a la acción que él tiene sobre ellos y que las relaciones causa-efecto requieren de un contacto físico y espacio. (Piaget, et ál., 2015).

En ese sentido, es necesario proporcionar a Nicolás un contexto oportuno para desarrollar las capacidades anteriormente mencionadas. Según Harlen (2007) cualquier modelo de la forma de aprender de los niños no es más que una hipótesis. Por su parte, Furman (2018) sostiene que "la enseñanza para la diversidad enfatiza la necesidad de que todos aprendan en profundidad, pero al mismo tiempo logren desarrollar su autonomía como aprendices y aprendan a elegir" (p.42). Por lo tanto, es necesario proporcionar a Nicolás la suficiente libertad para decidir cómo aprender y reflexionar sobre su propio proceso, sin perder de vista los propósitos previamente establecidos, potenciar las habilidades científicas y el lenguaje verbal. Esto sustenta también el anhelo por encontrar como docentes diversas maneras de aproximarnos a cómo ellos aprenden desde la práctica. Sin embargo, algo de lo que sí estamos convencidos es que, es a través de la experiencia que se forman las ideas.

Una de las principales áreas de conocimiento es aquella referida a la diversidad y características de los seres vivos y los procesos de la vida. Por otra parte, los procesos de la ciencia en la que se pretendía involucrar a Nicolás fueron la observación, la elaboración de predicciones, el planteamiento de preguntas, el registro de datos, la comunicación de resultados, entre otros. Es decir, los mismos procesos en los que se involucrarían los estudiantes sin características diferentes, solo que con estrategias de acuerdo con sus demandas y necesidades.

Los planteamientos fundamentales de diferentes pedagogos del siglo xx, en especial de Dewey, nos orientan a seguir sosteniendo que los niños pueden y necesitan involucrarse en procesos de investigación por ellos mismos. Partimos de este planteamiento con el propósito de involucramos con Nicolás en su propia experiencia científica, confiados en que el acercamiento guiado hacia un fenómeno natural que goza de la predilección de Nicolás, nos daría carta abierta al planteamiento progresivo de estrategias pedagógicas que permitan ir desarrollando su gusto por la ciencia y por comprenderla; pues es necesario redefinir el rol de la adquisición de conocimiento científico y entenderlo como aquella actividad que responde simplemente a la voluntad por comprender y satisfacer ese impulso tan natural y enriquecedor de nosotros, los seres humanos (Martí, 2012).

\section{Una expresión de curiosidad, una oportunidad para empezar a aprender ciencias}

Específicamente para la clase de Ciencias, Armstrong (2015) recomienda involucrar a los estudiantes en actividades al aire libre, de jardinería con aprendizaje basado en proyectos. El trabajo en actividades relacionadas con las ciencias supone también el trabajo en el área del lenguaje verbal dado que la expresión verbal sobre los fenómenos es el primer paso para ordenar un pensamiento y comprenderlo (Díaz, 2016). Es así como un sábado por la mañana mientras Nicolás exploraba en los cajones de la estantería de la cocina de su casa, una bolsa de menestras llamó su atención lo suficiente como para empezar a ordenar un puñado de estas de tal manera que cupieran en el posavasos de su pequeña mesa de trabajo. Nicolás tenía predilección por comunicarse a través de dibujos, por ello, enseguida, tomó una hoja y empezó a dibujar una de las semillas, la más grande (Figura 2). La exploración de sus ideas previas se inició con una simple pregunta 
qué, incluso él planteó para sí mismo y con una amplia sonrisa la pregunta "¿qué es esto?", que desencadenó una serie de eventos correspondientes a la experiencia que da lugar a este caso.

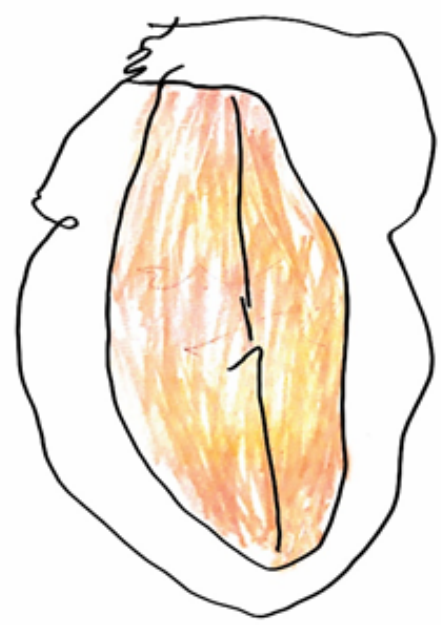

Figura 2. Dibujo de semilla de frejol realizado por Nicolás

\section{Dialogar para construir aprendizajes}

A la edad de dos años Nicolás no realizaba contacto visual con las personas que lo rodeaban y sus relaciones interpersonales se enfocaban en suplir sus necesidades. Luego de un arduo trabajo, mediado por actividades lúdicas, Nicolás ahora tiene una sorprendente capacidad para inferir información a partir de las emociones que expresan la persona con las que se encuentra relacionándose. A medida que Nicolás dibujaba, también observaba mi rostro expresándole alegría y aceptación; luego de dibujar, colorear y pegar su dibujo sobre la pizarra, retomé su pregunta de diciéndole ¿qué crees que es esto?, él dijo: "es una planta”. Entonces le pregunté "¿cómo sabes que es una planta?", con el fin de guiarlo en la elaboración de predicciones basadas en su experiencia (Harlen, 2007); a lo que Nicolás respondió: "el frejol crece si lo pones con tierra, agua y se lo pone al Sol".

Piaget planteó que los niños, en su necesidad de explicar la realidad, formulan preguntas e hipótesis en función de las regularidades observadas en esta misma realidad, y razón no le faltaba. Nicolás había visto algunos libros sobre el crecimiento de las plantas en la biblioteca familiar, otras plantas en el parque y en el jardín de casa. Aproveché ese momento para promover en él la verbalización de más elementos que formaban parte de su predicción, mientras estaba sentada frente a él en su pequeña mesa y podía visualizar mi rostro a su altura y a $50 \mathrm{~cm}$ de distancia. El trabajo frecuente con Nicolás había permitido la identificación de expresiones gestuales o señas propias de él, por lo que yo las utilizaba como andamios para lograr una mejor comprensión del lenguaje verbal. A continuación, se presenta la transcripción del diálogo sostenido:

Yo: ¿Y cómo vamos a hacer que crezca? [Seña de crecimiento]

Nicolás: La ponemos en la tierra y ahí echamos agua y la ponemos en el Sol. [Mientras realiza otro dibujo (Figura 3)]

Yo:¿Cuántos frejoles usarás? [Seña de contar, señalando los frejoles]

Nicolás: Uno.

Yo:¿Cómo le vamos a echar el agua? [Seña de echar agua]

Nicolás: Con una vasito. [Nicolás corrió a traer un pequeño vaso medidor de jarabes que había en la cocina]

Yo:¿Cuántos de estos [con el vasito medidor en la mano] le echaremos? [Seña de contar]

Nicolás:Un poquito, solo uno.

Yo:¿Dónde pondremos el frejol? [Seña de dónde]

Nicolás: Arriba, con el Sol.
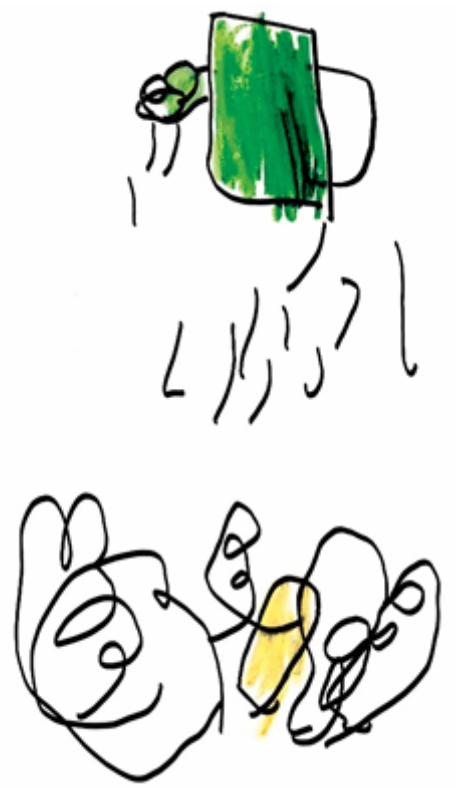

Figura 3. Dibujo realizado por Nicolás para explicar cómo sembrará la semilla de frejol 
Es importante mencionar que durante el trabajo pedagógico que se realizó con Nicolás, en todo momento se modeló emoción y respeto por el ambiente natural, además de la apertura a las posibilidades de experimentación que él planteaba; de acuerdo con Talhelm (2013), cuando se realiza este tipo de modelización estamos intencionalmente emitiendo el mensaje de que es este tipo de comportamiento el que esperamos y - tratándose de un niño cuyo modo de comunicación predilecto se encuentra en las imágenes con tipología de pictogramas-, la modelización era indispensable.

Shanker (2013) manifiesta que desde pequeños es necesario que los niños sean captados por su tutor primario, para involucrarlos y centrar su atención. En ese sentido, se deben ofrecer actividades cautivadoras, cuya vía predilecta sea el contacto visual amistoso, el tacto y la vocalización. Estas vías fueron nuestra ruta de comunicación continua con Nicolás; ello, sumado a su curiosidad natural, nos aseguró la atención durante las maravillosas semanas en las que aprendimos juntos.

Adicional a lo anteriormente expresado, en todo momento se acompañó la indicación verbal con el lenguaje gestual, además de una especie de simbología con las manos, establecida con él como andamio no verbal a las indicaciones que le dábamos. Se optó por elegir palabras cortas y previamente conocidas por él, pronunciadas tratando de focalizar la mirada de Nicolás en mi rostro. Así, por ejemplo si esperábamos que dibuje, se lo pedíamos, pero señalando los colores, la libreta de notas y el objeto a dibujar, aunque usualmente esta acción era de su interés y la petición no era siempre necesaria. El lenguaje verbal es un instrumento muy valioso, sin embargo, de acuerdo con la edad y al diagnóstico de Nicolás, su nivel de comprensión de este demandó modificar el lenguaje que usábamos, pues prevaleció la comprensión de cómo crece la semilla de frejol y la representación de esta, ya que solo se puede representar algo que se ha comprendido (Piaget, en Labinowicz, 1980).

\section{Explorar libremente para expresar verbalmente}

De acuerdo con Furman y De Podestá (2013), la experiencia concreta aporta algo realmente valioso a la hora de desarrollar el gusto por explorar el mundo que nos rodea y construir nuestro propio conocimiento sobre el mismo: el despertar nuestra necesidad de plantear preguntas, y así expandir cada vez más nuestra necesidad de experimentar para responderlas. Para cuando, paulatinamente, se le presentaron a Nicolás otros materiales como algodón, agua, vaso descartable, el rápidamente envolvió al frejol en algodón y lo humedeció, retirando el excedente de agua para que, en sus palabras "no se ahogue"; además, se dirigió rápidamente al cuarto de estudio de la casa para traer una lupa y observar cada detalle de la semilla colocada en el algodón (Figura 4); también tuvo especial cuidado de colocar la semilla exactamente en el centro del recipiente.

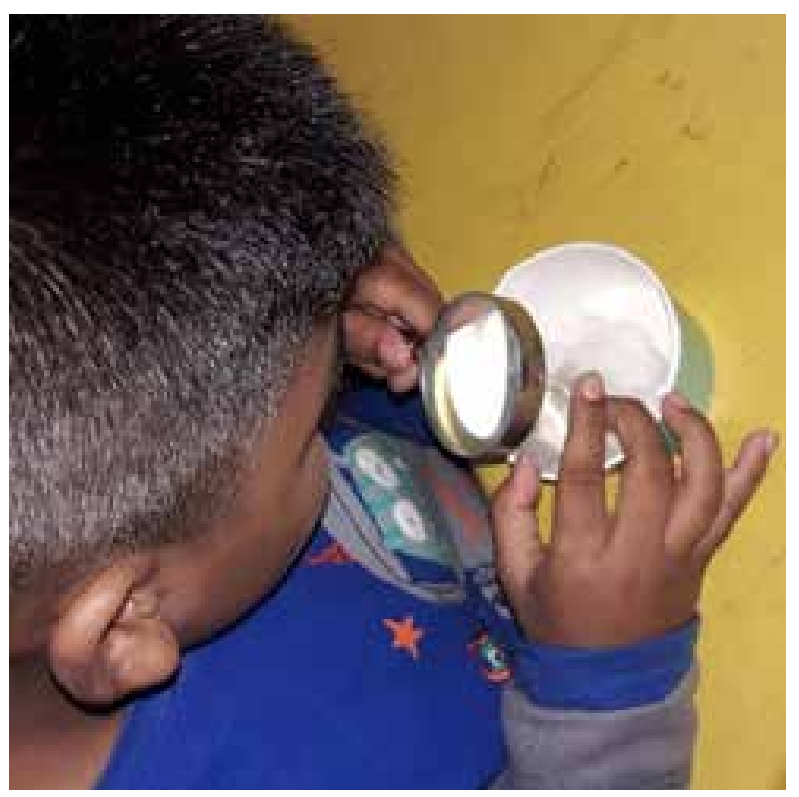

Figura 4. Nicolás y su uso espontáneo de la lupa

Al caer la tarde soleada y, como previamente habíamos conversado, Nicolás subió hacia la azotea y colocó el vaso con la semilla en un lugar donde le daban los rayos de sol diciendo "para que la semilla de frejol crezca". Nicolás se aseguró, sin indicación alguna, de que el vaso quede expuesto al sol y esté lo suficientemente humedecido. Desde ese momento nos sumergiríamos en un minucioso, paciente y largo proceso de observación, pues Nicolás necesitaba comprobar su predicción: "la semilla de frejol crecería si la ponemos en la tierra, se le echa agua y la ponemos al sol", el detalle es que Nicolás pensaba que esta semilla crecería muy pero muy pronto. Es usual que las hipótesis planteadas por los niños sean contrarias a lo que a los adultos nos parece evidente, no obstante, lo realmente enriquecedor es el proceso de someterlas a prueba para que se genere un conflicto $y$, a partir de la observación de un determinado evento o fenómeno, comience la construcción de nuevas ideas.

\section{Contar lo que experimentas genera evidencias de aprendizaje}

De acuerdo con Charpak (2005), los experimentos divierten, pero deben conducir al logro de descubrimientos 
efectivos, por lo que las actividades de manipulación de materiales deben estar apoyadas en preguntas que permitan a los niños proponer posibles explicaciones, de tal manera de que se vayan construyendo las nociones. Nociones que por cierto no necesariamente son las que los docentes pretendemos que sean, sino que se precisa de un trabajo de investigación para poder identificar esos temas, cuyo conocimiento es susceptible de ser construido por los niños.

El niño construye sus primeros aprendizajes sobre los fenómenos naturales a medida que explora el mundo que lo rodea, es principalmente su curiosidad innata la que lo moviliza a indagar su entorno.

Esa noche Nicolás manifestó su anhelo de dormir pronto, pues quería ver, al día siguiente la hermosa planta en la que el frejol se había convertido.

Y para atender a esta idea y desarrollar la noción de temporalidad aprovechamos el conocimiento que tenía Nicolás sobre la representación formal de los números y elaboramos una tabla en la que él iría marcando los días que transcurrirían en el crecimiento del frejol (Figura 5). Así mismo, establecimos que cada día después de almuerzo, iríamos a observar lo que había pasado con la semilla de frejol. Es bastante claro que él prefería ver para creer y esto era lo pedagógicamente oportuno.

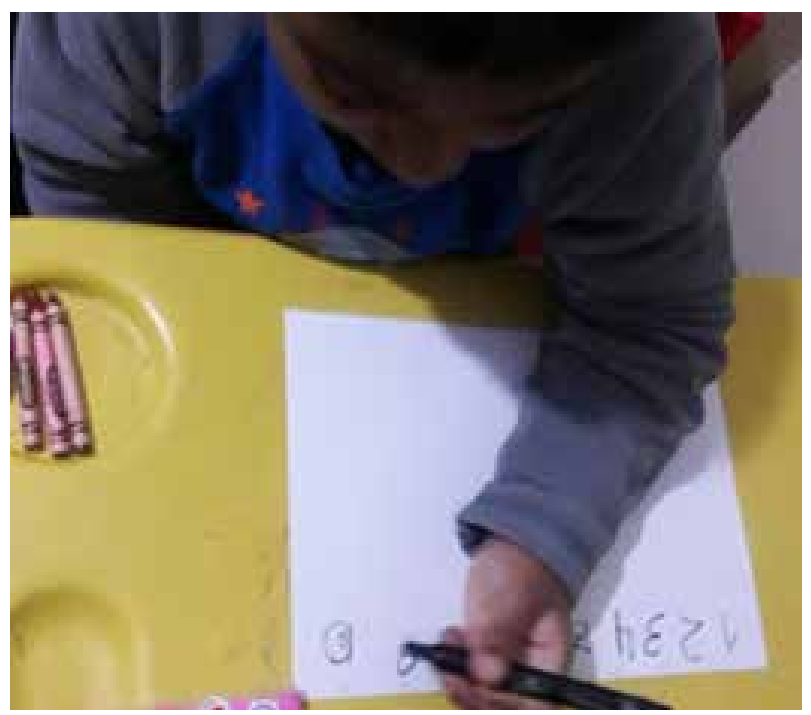

Figura 5. Tabla construida para el registro de datos

Al día siguiente, Nicolás despertó muy temprano y presuroso se dirigió hacia la azotea de la casa para poder visualizar lo que él creía que iba a pasar. Al notar que la semilla de frejol no había crecido, lo invité a mirar todas las macetas que la familia solía cultivar y le pregunté “¿Cuándo crees que crecerá la semilla?”; Nicolás predijo sonriente y con la mano en la cabeza, dijo: "en diez días". Según Harlen (2007) la predicción se puede basar en una pauta detectada en las observaciones, y así fue, ya que Nicolás había vivenciado, junto con algunos integrantes de la familia, que las plantas que riegan frecuentemente en la azotea no crecen de un día para otro. Su sonrisa contagiante y cierto aire de complicidad manifestado en su rostro nos daban evidencia para pensar que efectivamente había identificado dicha pauta a partir de estas vivencias familiares.

Nicolás, estuvo pendiente del crecimiento de la semilla de frejol en las próximas horas e incluso se percibió que su afán se intensificaba cuando hacía más calor durante el resto del día. En los próximos días, la frecuencia de las veces en las que Nicolás visualizaba el vasito con la semilla de frejol se fue reduciendo y su principal interés cambió hacia mantener el algodón húmedo constantemente, él decía: "porque la semilla tiene sed, hace mucho calor”.

Para la mañana en la que se avizoraron los primeros cambios en la semilla de frejol, Nicolás no podía contener la alegría, su rostro denotaba ternura y satisfacción al ver la pequeña rádula que emergía del frejol, emocionado se dispuso a observar con la lupa los cambios que se habían dado en dicha semilla.

Entonces me centré en que acompañé sus observaciones con dibujos (Figura 6); guiamos su atención hacia los detalles que él no notaba pero que, aprovechábamos como excelente oportunidad para desarrollar su oralidad en relación con lo que estaba observando. Usamos la regla -Nicolás gustaba mucho de observar los números de la regla y de medir cosas-, no fue necesario plantearle indicación alguna para que las use y registre el número hasta donde llegaba la pequeña planta en crecimiento y así fue día a día, aún sin saber las unidades de medida de la regla él identificó el cambio en el tamaño a partir de unas marcas que hicimos con plumones de colores en la misma regla. En la tabla previamente construida, Nicolás utilizó un aspa para marcar el día en el que la semilla creció. Así mismo, cada vez que Nicolás regaba la semilla con el pequeño vaso medidor, dirigía su atención hacia aquellos números que venían grabados en dicho vasito, yo le preguntaba "¿está bien la cantidad de agua que le has echado?”, a lo que Nicolás fue encontrando la regularidad para decir que cuando era mucha agua el algodón quedaba demasiado humedecido y el agua salía de él y, que era poca agua cuando el algodón no se humedecía en gran parte. 
Día tras día Nicolás observaba con una lupa y dibujaba con detalle lo que pasaba con el frejol. Su rostro de sorpresa al observar que poco a poco los cotiledones de lo que fue en algún momento la semilla de frejol, quedaron suspendidos en él, ahora, tallo con hojas (Figura7). Con el transcurrir de los días Nicolás dijo: "la semilla de frejol demora bastante en crecer, pero crece bien bonita y cada día un poco más". Aproveché esta oportunidad para observar la hoja de registro del crecimiento de la semilla que Nicolás había diseñado en el día 1 y le pregunté “¿en cuántos días cambió el frejol?". Apoyé esta pregunta con

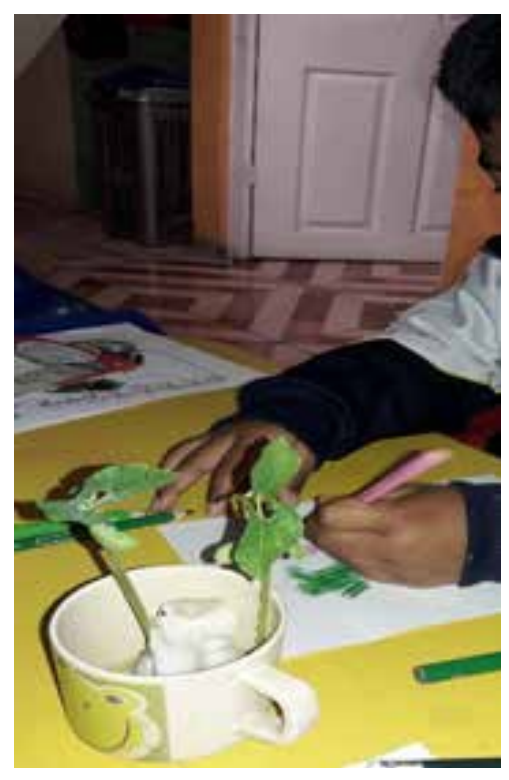

Figura 6. Nicolás dibujando el crecimiento del frejol semilla

\section{A modo de conclusión}

Una de las cosas que más disfruto en la vida es enseñar a amar las ciencias naturales. Esto es innato en mí, no me es necesario realizar esfuerzo alguno para declararlo abiertamente puesto que mi predilección, es demás evidente. Otra de las cosas que me apasiona es ver cómo se pueden ir descubriendo oportunidades de aprender a medida que aprendes de manera conjunta, con los niños. Esto último es una maravillosa y, hasta cierto punto, exclusiva oportunidad de aquellos que nos dedicamos a la docencia.

Las posibilidades de hacer investigación-acción son múltiples en nuestro campo de trabajo y realmente es necesario aprovecharlas para reafirmar la identidad de nuestra labor. Sabemos que existe un cuerpo teórico bastante extenso y sólido que orienta nuestra práctica las señas usuales a modo de andamios para favorecer la comprensión de la pregunta. Él respondió: "crece en ocho días". Nicolás había descubierto esto por sí mismo y, como bien advertía Mayo (en Rivero, Martín, Solís y Porlán, 2017), no se le explicó lo que él pudo descubrir por sí mismo. Esta aventura empezó con el propósito de responder principalmente a la curiosidad natural de Nicolás, la cual se fue expresando paulatinamente en predicciones sobre lo que necesita una semilla de frejol para crecer y la temporalidad en la que se daría su primer brote.

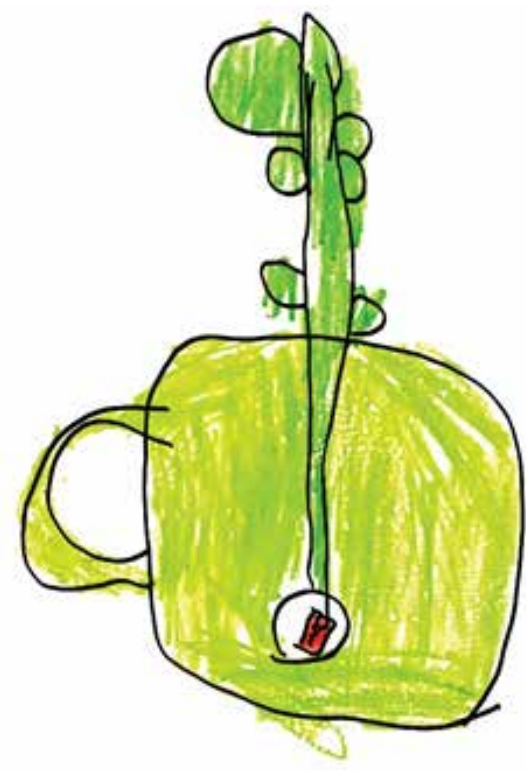

Figura 7. La planta de frejol en el día 12

pedagógica, pero también sabemos que pertenecemos a realidades totalmente diversas y que dichas realidades nos plantean retos todo el tiempo. Es en ellas donde reinventamos la teoría. Es fascinante la manera en la que podemos proveer oportunidades de aprendizaje, de acuerdo con las demandas de los estudiantes y promover logros en ellos, pero, algo aún más fascinante, es generar aprendizajes donde inoportunamente alguien dijo que no se iban a lograr y tener el placer de aprender de lo que hacemos.

La puerta de entrada al aprendizaje es la empatía y el vínculo afectivo que se establece entre dos personas con amplias ansias de aprender, por lo que recomiendo que, de buenas a primeras no nos embarquemos en determinación de objetivos académicos de acuerdo con estándares de aprendizaje, sin previamente haber formado ese vínculo que nos dará el boleto al viaje completo. 
En mi experiencia con Nicolás, ambos éramos realmente cómplices y ávidos por ver cómo crecía la semilla de frejol. Las investigaciones que se realizan con las semillas de frejol son un manera muy sencilla y valiosa de hacer indagaciones respecto, por ejemplo, a las condiciones que favorecen y controlan su crecimiento, la velocidad de este, la comparación del tiempo de crecimiento frente al de otras semillas, entre otras (Friedl, 2005); y, aunque yo sabía sobre las semillas de frejol lo que él no, algo que realmente yo no sabía, era cómo respondería un niño que aparentemente no toleraba contacto con texturas como la tierra, el barro, el musgo, cuando trabajáramos con ello.

A partir de esta experiencia hay un sinfín de otras maravillosas oportunidades que seguramente, a medida que Nicolás crezca, iremos viviendo. Esta complicidad que habíamos ya establecido es también la que nos lleva actualmente por ejemplo, a explorar el interior de los tomates. Juntos nos embarcamos cada vez en tareas más grandes y ambiciosas, donde características como su excesivo movimiento e intranquilidad, enriquecen el trabajo en la azotea de la casa y favorecen que Nicolás tome trascendentales decisiones, como el ahondar sus brazos en la mezcla de tierra con abono, mientras yo lo acompaño en su decisión con una clara aprobación hacia lo que sea que decida, pero siempre con altas expectativas de lo que él puede hacer, ya sea que comulgue con lo que yo tenía pensado o no. En esta ocasión tengo licencia para aseverar que un gesto vale más que mil palabras y esto no es negociable en nuestra labor docente ni en casa.

A partir de este tiempo en el que Nicolás y yo nos hicimos cómplices en la búsqueda de respuestas, su pregunta favorita fue ¿qué pasará? Es así, que abrimos frutos y sembramos sus semillas para ver ¿qué pasará si las sembramos como lo hicimos con los frejoles? Y todo esto frente a una sonrisa que se expresa entre la contagiosa curiosidad y la decisión propia de tocar algo que no estaba antes dispuesto a tocar. Noto cómo Nicolás se esfuerza por verbalizar frases para expresar lo que siente, lo que piensa que iba a pasar, pero ahora se evidencian frases más largas y fluidas que al inicio.

De acuerdo con Furman y de Podestá (2013) la observación y la descripción son algunas de las competencias fundamentales durante la exploración de un determinado fenómeno, además pueden desarrollarse desde los primeros años de la escuela primaria. Es por demás evidente que de acuerdo con la experiencia, el punto de partida de estas competencias es tan potencialmente natural y susceptible de ser promovido desde incluso edades más tempranas; de igual forma, la exploración de los fenómenos es un vehículo privilegiado para desarrollar la oralidad en niños como Nicolás, puesto que es muy significativo hablar de lo que te sorprende y genera curiosidad.

Nicolás asumió el reto que le planteaba el expresarse en frases para poder manifestar lo que observaba, todo eso gracias a su curiosidad natural, algo en lo que yo no estuve involucrada, pero que aproveché de la manera en la que ya se los he contado. Gracias a la curiosidad natural de Nicolás, en los próximos días, semanas y meses nos vimos inmersos en preguntas de "qué pasará si" y, en la medida de lo posible, juntos buscamos respuestas. Así, hemos pasado momentos inolvidables observando la cara de la luna, corroborando información en los calendarios que aun consideran la fase de la Luna que se observará en el cielo, abriendo frutos, mezclando cosas, viviendo la ciencia.

Es preciso reafirmar la necesidad de generar oportunidades de aprendizaje que respondan a las características y demandas de los estudiantes, puesto que dichas oportunidades se constituyen como espacios en los que el estudiante construye conocimientos integrales, no solo respecto del fenómeno que se dispone a estudiar, sino que, también construye aprendizajes respecto de sí mismo. Asimismo, en edades tempranas construye la percepción sobre sí mismo, lo que puede hacer, sus potencialidades y lo que necesita para alcanzar retos que le plantea su propio ser y el mundo que lo rodea. Nosotros los docentes tenemos esta compleja pero a la vez fascinante labor, que atraviesa por también ir descubriendo lo mucho que podemos hacer para responder a dichas necesidades y demandas a fin de profesionalizarnos cada día más.

\section{Referencias}

American Psychiatric Association. (2013). Diagnostic and Statistical Manual of Mental Disorders. Fifth Edition. Autoedition.

Armstrong, T. (2015). Neurodiversidad en el salón de clases. Estrategias basadas en fortalezas que ayudan a los alumnos con necesidades especiales a tener éxito en la escuela y en la vida. Lima: Cerebrum ediciones.

Berlyne, D. (1954). A theory of Human Ciosity. British Journal of Psychology, 45(3), 180-191.

Centro Peruano de Audición, Lenguaje y Aprendizaje (CPAL). (2019). Bases teóricas de las dificultades especificas de aprendizaje. Material del curso Dificultades Específicas de Aprendizaje: Desafíos en la Escuela. Lima: CPAL-Escuela de Estudios Superiores. 
Cerebum, Neurociencias, Educación y Desarrollo Humano. (2014). Todos y cada uno de nosotros frente al aprendizaje: cerebros únicos e irrepetibles, módulo 14 y 15. Programa Brainbox. Autoedición.

Charpak, G. (2005). Manos a la obra. Las ciencias en la escuela primaria. México: Fondo Educativo de cultura económica.

Congreso de la República del Perú. (2018, 21 de junio). Ley que promueve la educación inclusiva, modifica el artículo 52 e incorpora los artículos 19-a y 62 -a en la ley 28044, ley general de educación. El peruano. https://busquedas.elperuano.pe/download/url/ley-que-promueve-la-educacion-inclusiva-modifica-el-articul-ley-n-30797-1662055-2

Díaz, C. (2016). Estructuras didácticas para Ciencias Naturales. Estrategias integradoras para explorar, experimentar e investigar. Buenos Aires: Ediciones Novedades Educativas.

Dewey, J. (2007). Cómo pensamos. La relación entre pensamiento reflexivo y proceso educativo. España: Paidós.

Friedl, A. (2000). Enseñar ciencias a los niños. España: Gedisa.

Furman, M. (2018). Guía para criar hijos curiosos. Ideas para encender la chispa del aprendizaje en casa. Buenos Aires: Siglo XXI editores.

Furman, M. y De Podestá. (2013). La aventura de enseñar ciencias naturales. Buenos Aires: Aique.

Harlen, W. (2007). Enseñanza y aprendizaje de las ciencias. Madrid: Morata.

Hornok, J. (2018). United in Autism. Finding Strength inside the Spectrum. Dallas: Brown Books Publishing Group.

Labinowicz, E. (1980). Introducción a Piaget. Pensamiento, aprendizaje, enseñanza. España: Fondo Educativo Interamericano.
Kashdan, T., Steger, M. y Breen, W. (2007). Curiosity. https://www.researchgate.net/ publication/232709051_Curiosity/download

Kidd, C. y Hayden, B. (2015). The Psychology and Neuroscience of Curiosity. Neuron, Perspective, 88, 449-460. http://dx.doi.org/10.1016/j. neuron.2015.09.010

Martí, J. (2012). Aprender Ciencias en la Educación Primaria. Barcelona: Graó.

Ministerio de Educación del Perú. (2011). Normas técnicas para el diseño de locales de educación básica regular. Autoedición. http://www.minedu.gob.pe/ files/107_201109011135.pdf

Ministerio de Educación del Perú. (2017). Currículo Nacional de la Educación Básica. Autoedición. http:// www.minedu.gob.pe/curriculo/

Piaget, J. y Inhelder, B. (2015). Psicología del niño. Madrid: Morata.

Rivero, A., Martín, R., Solís, E. y Porlán, R. (2017). Didáctica de las ciencias experimentales en educación primaria. Madrid: Síntesis.

Rowson, J. (2012). The Power of Curiosity. How linking inquisitiveness to innovation could help to address our energy challenges. London: RSA Social Brain Centre.

Shanker, S. (2013). Calma, atención y aprendizaje. Estrategias para la autorregulación en el aula. Lima: Cerebrum.

Talhelm, M. (2013). Starting with Science. Strategies for Introducing young children to inquiery. Portsmouth: Stenhouse Publishers.

Vega. S. (2012). Ciencia 3-6. Laboratorios de ciencias en la escuela infantil. Barcelona: Graó. 